\title{
Sucking pressure and its relationship to milk transfer during breastfeeding in humans
}

\author{
C. R. Prieto ${ }^{1}$, H. Cardenas ${ }^{2}$, A. M. Salvatierra ${ }^{1}$, C. Boza ${ }^{3}$, \\ C. G. Montes ${ }^{3}$ and H. B. Croxatto ${ }^{3}$ \\ ${ }^{1}$ Instituto Chileno de Medicina Reproductiva, Casilla 96, Correo 22, Santiago, Chile; ${ }^{2}$ Departamento de \\ Ciencias Biológicas, Universidad de Santiago de Chile, Casilla 307, Correo 2, Santiago, Chile; and \\ ${ }^{3}$ Unidad de Reproducción y Desarrollo, Universidad Católica de Chile. Facultad de Ciencias Biológicas, \\ Casilla 114-D, Santiago, Chile
}

\begin{abstract}
Breast sucking pressure has been only partially characterized in humans and its quantitative relationships with milk transfer and endocrine maternal responses are unknown. A method to record sucking pressure and milk transfer during complete sucking episodes is described. A tubing connected at one end to a pressure transducer was attached to the nipple so that the baby sucked both the nipple and the catheter during breastfeeding. The transducer's signals were fed into a commercial computer system designed to digitize and analyse physiological signals. A total of 27 recordings, 13 of which were from a single breast and 14 from both breasts were evaluated. Average values for the mean and maximum sucking pressures were -50 and $-197 \mathrm{mmHg}$, respectively; the median intersuck interval was $0.7 \mathrm{~s}$; and duration of the sucking episode was $7 \mathrm{~min}$. Diverse sucking pressure patterns were observed due to different mixes of sucking bursts with steady sucking and stable versus decreasing pressure and frequency throughout each sucking episode. The amount of milk transferred to the baby was estimated from the difference in body weight immediately before and after each episode. Milk transfer from the second breast was 58\% lower than from the first; this was associated with a significant decrease in grams of milk transferred per suck or per minute without significant changes in sucking pressure. The data suggest that there is a change in the maternal physiological response to sucking between the first and second breast. This report shows the feasibility of measuring the sucking pressure developed by human babies during complete nursing episodes, and offers great potential to explore the relationships between the physical parameters of sucking and maternal physiological responses, such as hormonal changes, milk yield and duration of lactational amenorrhoea.
\end{abstract}

\section{Introduction}

Breastfeeding has been the subject of numerous studies on account of its nutritional and protective health values for the newborn. Breast sucking is a key component of breastfeeding since it provides the mechanical basis for the transfer of milk from the breast to the infant. In addition, it stimulates mechanoreceptors in the nipple and areola, triggering neuroendocrine responses that play a central role in maternal physiology during lactation, including the extension of postpartum amenorrhoea.

Ardran et al. (1958a, b) described the movements of the baby's mouth during breast sucking using $X$-ray imaging and suggested that the human baby obtained the milk from the mother's breast mainly by compression of the bulb of the teat, by a squeezing action of the tongue, rather than by sucking. However, direct recordings from the mouth during sucking revealed the development of important negative pressures that

Received 5 February 1996. may have a role in the extraction of milk from the breast (Colley and Creamer, 1958; Kron et al., 1962). The negative pressure may serve also to secure a firm attachment to the breast while compressing the bulb. As stated by Wakerley et al. (1994), the relative contributions of intra-oral negative pressure and of nipple compression in milk transfer are still controversial.

Whatever its role in milk transfer, the frequency and intensity of sucking may be closely related to the amount of stimulation received by the mechanoreceptors of the nipple and areola. If this is the case, significant correlations could be expected between quantitative parameters of the sucking activity developed throughout a nursing episode and acute maternal responses, such as the ensuing prolactin surge. Similarly, the product of intensity of sucking and the total time spent by the baby at the breast in $24 \mathrm{~h}$ may determine the amount of sensory stimulation of the breast on a daily basis and the duration of amenorrhoea. A positive correlation has been found between number and duration of breastfeeding episodes per day and the duration of amenorrhoea (Howie 
et al., 1982; Díaz et al., 1988); however, this correlation is not strong owing to large inter-individual variation in the duration of amenorrhoea among subjects with similar breastfeeding patterns. It is possible that by including in the equation the intensity of sucking at each episode such correlation may become stronger.

Systematic studies of the sucking pressures during breastfeeding and its relationship to milk transfer in the human baby, as well as to the duration of amenorrhoea, have not been carried out, probably owing to the lack of an appropriate pressure recording method that does not disturb breastfeeding, and also the lack of a practical tool for the quantitative analysis of such recordings. In the present paper, we describe a relatively simple method to record and analyse the intra-oral negative pressures developed by human babies during breast feeding, and preliminary data on the quantitative relationship of various parameters of sucking activity with the amount of milk transferred are given.

\section{Materials and Methods}

\section{Subjects}

The data presented were obtained from 17 mothers recruited at the Family Planning Clinic of the Instituto Chileno de Medicina Reproductiva. The study protocol was approved by the Ethics Committee and informed consent was obtained from each mother. All had had a normal pregnancy, vaginal delivery at term, normal birth weight and normal postpartum evolution. At the time sucking pressure was recorded, they were fully breastfeeding on demand six or more nursing episodes per day. Breast milk was the only source of water and nutrients for the babies up to 6 months of age. From 7 months of age, non-dairy supplements were given. Breastfeeding patterns and social habits of this population have been previously described (Diaz et al., 1988). None of the mothers had used hormonal contraceptives since delivery. The group was purposely heterogeneous in terms of age and weight of mothers and babies in order to test the recording system within a wide range. The ages of the individual babies included in this report ranged from 21 to 240 days, and their weights from $4205 \mathrm{~g}$ to more than $8000 \mathrm{~g}$ (Table 1).

\section{Recording sucking pressure}

In this report, a sucking episode is defined as the entire period during which the baby is attached to a nipple until definite detachment is initiated by the baby or the mother, and which in the mother's judgement corresponds to her usual breastfeeding practice. When sucking episodes were recorded from both breasts, they are noted as one nursing episode. The choice of the first breast was always made by the mother. All recordings were made between 12:00 and 15:00 h at least $2 \mathrm{~h}$ after the preceding breastfeeding episode.

Thirteen sucking episodes from a single breast were recorded in ten subjects and one nursing episode was recorded for seven subjects. Of the 17 mother-baby pairs studied, 10, 5, 1 and 1 contributed with a recording once, twice, three and four times, respectively. Most of the time, the catheter in the
Table 1. Age and body weight of the babies at the time of the first recording

\begin{tabular}{lc}
\hline Age (days) & Body weight $(\mathrm{g})$ \\
\hline & \\
\hline 21 & 4205 \\
41 & 4570 \\
43 & 5915 \\
47 & 5220 \\
47 & 5025 \\
53 & 5760 \\
56 & 5775 \\
57 & 6147 \\
90 & 6216 \\
120 & 7580 \\
129 & 7040 \\
180 & 8249 \\
183 & 8959 \\
184 & 7235 \\
200 & 7946 \\
240 & 8378 \\
\hline
\end{tabular}

The age of one child was not available and one older baby could not be weighed because he did not fit in the weighing balance.

nipple was ignored by the babies and according to the mother's opinion breastfeeding during recordings was indistinguishable from that occurring normally. However, some babies older than 6 months exhibited curiosity about the catheter and this disturbed their breastfeeding. These recordings were excluded from the analysis.

A Silastic tube $(15 \mathrm{~cm}, 0.16 \mathrm{~cm} \quad$ i.d. $\times 0.24 \mathrm{~cm} \quad$ o.d.; Konigsberg Instruments Inc., Pasadena, CA) was taped over the mother's breast with its tip at the level of the nipple, while the other end was connected to a polyethylene catheter (PE 90). Both tubes were filled with sterile water. The polyethylene tubing (length $85 \mathrm{~cm}$ ) was connected to a pressure transducer (P23, Statham Medical Instruments Inc., Hato Rey, Puerto Rico) which was plugged into a preamplifier (Vorverst TF, Hellige $\mathrm{GMBH}$, Freiburg). The signal from the preamplifier was digitized using the Mac Pacq 10 Work Station System (BioPac Systems, Goleta, CA), and stored in a Macintosh LC II computer for further analysis by the Acq Knowledge software (V 2.0.10, BioPac Systems).

The digitized recordings allowed easy quantification of several parameters by the Acq Knowledge software: the duration of the sucking episode, the mean and maximum pressures, the area under the curve of the pressure changes and the total number of sucks.

The intersuck intervals are not easy to obtain in the case of long recordings exhibiting variations in baseline. The pressure recordings were therefore first submitted to band pass filtering using the Acq Knowledge software. By trial and error, it was found that after setting the low and high frequencies of the band pass at $1 \mathrm{~Hz}$ and $4 \mathrm{~Hz}$, respectively, the pressure changes of all sucks passed through 0 , making it possible to compute the intersuck intervals. After storing the series of intersuck intervals in ASCII format they were statistically described using Statistica/Mac Software rel. 3. (Stat Soft, Tulsa, OK). 
Before and after each sucking episode, the babies were weighed, wearing their clothing, on an electronic digital balance accurate to $0.1 \mathrm{~g}$ (Sartorius, model 3862). The total amount of milk transferred was estimated from the difference between both weights. The accuracy of this procedure was tested in an independent study, in which after recording the weight and before removing the baby from the balance, a calibrated weight, in the range $2-40 \mathrm{~g}$, was added to the balance unknown to the observer. The difference between expected and observed weight increment lay between -0.4 and $+0.2 \mathrm{~g}$ and was not statistically different from $0(n=11$ for each weight). However, the difference in weight, with or without the addition of a $2 \mathrm{~g}$ weight, was statistically significant in babies up to $7 \mathrm{~kg}(P<0.002, n=11)$. For heavier babies (up to $11 \mathrm{~kg}$ ) the addition of a $5 \mathrm{~g}$ weight was required to detect a statistically significant difference $(P<0.002, n=5)$. Each baby was also weighed naked to obtain the absolute body weight.

In addition to the basic parameters used to characterize the sucking activity, an attempt was made to estimate the efficiency of sucking for obtaining milk. The efficiency was estimated as the ratios of gain in body weight during a sucking episode over: (a) the duration of the episode $\left(g \min ^{-1}\right)$, (b) the area under the pressure curve $\left(\mathrm{g}(\mathrm{mmHg} \times \mathrm{min})^{-1}\right)$, and $(\mathrm{c})$ the total number of sucks in the episode ( $g$ per suck). These ratios were used to compare the efficiency of sucking from the first and second breast in a nursing episode.

\section{Statistical analyses}

Description of variables and paired statistical comparisons were made using the Statistica/Mac Software rel. 3. Values given in the text and tables are mean \pm SE unless stated otherwise.

\section{Results}

Twenty-seven sucking episodes were recorded from seventeen mothers and their babies. Ten recordings were obtained from the first breast and three from the second breast only. Recordings from the first and the second breast in the same nursing episode were obtained in seven cases.

The overall mean duration and pressure of 27 sucking episodes was $7.0 \pm 0.7 \mathrm{~min}$ and $-50 \pm 5.7 \mathrm{mmHg}$, respectively. Other parameters of sucking are described (Table 2).

Most of the recordings displayed a complex pattern. Recordings that lasted a longer time exhibited a noticeable decrease in sucking activity during the episode (Fig. 1). To quantify this decrease, recordings lasting more than 5 min were selected and the maximum and mean pressure, the intersuck intervals and the area under the curve of the first and second half of the sucking episode were compared (Table 3). The higher sucking activity of the first half was accounted for by a greater sucking pressure and shorter pauses which explains the important difference in area under the curve between both halves. When the same analysis was applied to recordings lasting a shorter time (5 $\mathrm{min}$ or less), no significant differences were found between the first and the second half.
Table 2. Overall sucking parameters

\begin{tabular}{lc}
\hline Duration of episode (min) & $7.0 \pm 0.7$ \\
Mean pressure $(\mathrm{mmHg})$ & $-50 \pm 5.7$ \\
Maximum pressure $(\mathrm{mmHg})$ & $-197 \pm 10$ \\
Area under the curve $(\mathrm{mmHg} \mathrm{min})$ & $270 \pm 3878$ \\
Median intersuck interval (s) & $0.7 \pm 0.1$ \\
Number of sucks & $364 \pm 183$ \\
Milk transfer & \\
$\quad$ total weight gain (g) & $56 \pm 7.8$ \\
grams min $^{-1}$ & $8.6 \pm 5.9$ \\
grams (mmHg min) $^{-1}$ & $0.33 \pm 0.04$ \\
grams per suck & $0.17 \pm 0.15$
\end{tabular}

Values are mean \pm SE of 27 recordings.

The other component of the pattern was the occurrence of bursts of sucking separated by periods with considerably less activity or no activity at all, which lasted from about $2 \mathrm{~s}$ to more than $15 \mathrm{~s}$. The number, frequency and duration of these pauses exhibited no regularity within individual episodes (Fig. ib). The intersuck interval was used to describe the frequency of sucking. Since intersuck intervals did not follow a normal distribution, they are described by the median and percentiles. No attempt was made at this time to differentiate intersuck from interburst intervals.

Recordings of nursing episodes involving both breasts were obtained in seven cases. The parameters of sucking corresponding to the first and the second breast are given (Table 4). There were no significant changes in the sucking parameters, including duration of the episode, but all the estimates of the sucking efficiency were lower in the second breast. The total weight gain (Wilcoxon matched pairs test, $P=0.043$ ), and the weight gain per minute $(P=0.043)$, per area under the sucking pressure curve $(P=0.028)$, and per suck $(P=0.028)$, all decreased by more than $50 \%$ in the second breast (Table 4).

\section{Discussion}

The data presented in this study demonstrate the feasibility of recording the sucking pressure produced by human babies up to 8 months old during normal breastfeeding episodes. The duration of each episode, the sucking frequency and the total amount of milk transferred during episodes in which the sucking pressure was recorded are comparable to those reported by others under non-recording conditions (see Drewett and Woolridge, 1979; Bowen-Jones et al., 1982; Woolridge et al., 1982a). Therefore, the recording procedure does not seem to interfere with normal breastfeeding, either objectively or subjectively according to the mothers perception. Recordings of sucking pressure of babies feeding on artificial devices have been reported (see Colley and Creamer, 1958; Kron et al., 1962; Hack et al., 1985), but comparison of those pressure data with ours is not meaningful since resistance to milk flow is unknown in both sets of data.

A common feature of all recordings was the occurrence of sucking bursts separated by short, irregularly spaced pauses that tended to be more prominent in the second half of the sucking episode. In addition, sucking pressure curves lasting 
(a)
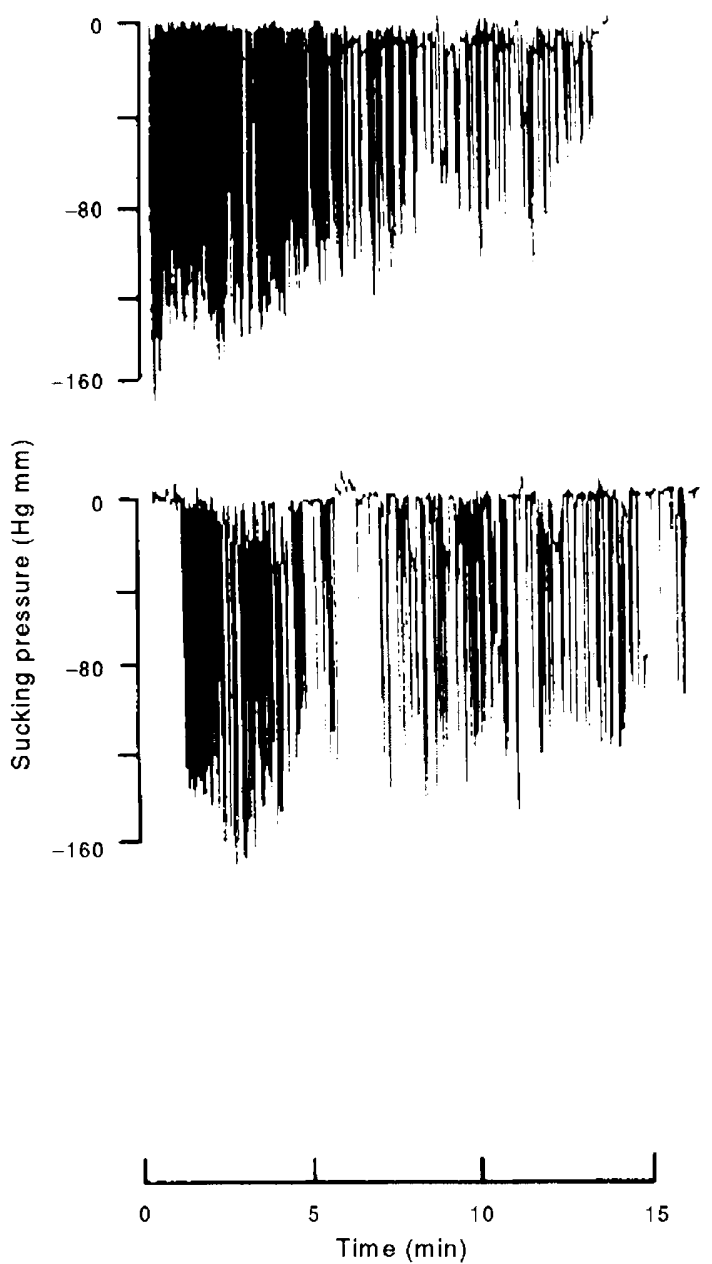

(b)
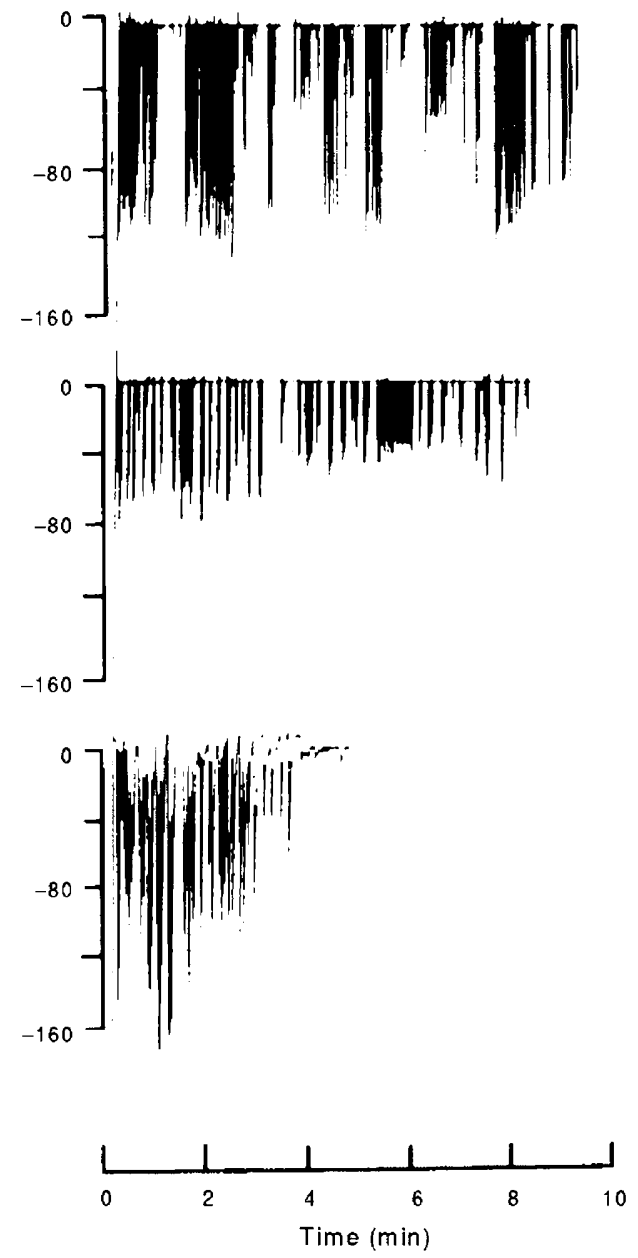

Fig. 1. (a) Continuous recording of sucking pressure during complete sucking episodes from the first breast for two pairs of mother and baby. The upper lane was obtained from a baby boy (5915 g bodyweight) who gained $152 \mathrm{~g}$ at the end of 14.6 min of sucking and the lower lane was obtained from a baby girl ( $5775 \mathrm{~g}$ bodyweight) who gained $55 \mathrm{~g}$ at the end of $16.3 \mathrm{~min}$ of sucking. Note the gradual decrease in frequency and amplitude along both recordings. (b) Individual sucking pressure curves of three babies who displayed bursts of sucking activity. Note the change in the time scale relative to (a). The upper lane illustrates the sucking of the second breast by a baby girl who gained $40 \mathrm{~g}$ during $10.4 \mathrm{~min}$ of sucking; the middle lane illustrates sucking of the first breast by a baby girl (bodyweight gain was not recorded) and the lower lane illustrates the sucking of the first breast by a baby girl who gained $76 \mathrm{~g}$ during 5.2 min of sucking. Compare with pattern displayed in (a).

more than 5 min were characterized by a decrease in sucking activity during the episode as indicated by a decrease in the amplitude of the negative pressure waves and in their frequency. Interestingly, the profiles of the rate of spontaneous milk flow from the breast, recorded with a miniature Doppler ultrasound transducer by Woolridge et al. (1982b), also displayed bursts of milk flow superimposed on a steady decrease during the sucking episode.

Our data confirm the decrease in total milk transfer during feeding from the second breast previously reported by Lucas et al. (1979) and Woolridge et al. (1982a). Possible explanations for the lower contribution of the second breast to total feeding are decreased sucking activity of the baby and/or a change in the maternal physiological response to sucking. None of the individual parameters of sucking activity investigated differed significantly between the two breasts; therefore, a difference in sucking activity does not explain the $58 \%$ fall in the amount of milk transferred. Moreover the sucking efficiency, expressed in grams of milk transferred per suck, or per minute, diminished to the same extent. It is possible, but it seems unlikely, that the squeezing action of the tongue decreased while sucking from the second breast, whilst at the same time the sucking activity remained unchanged. Nevertheless, the present data clearly demonstrate that the amount of milk transferred during a complete sucking episode is not determined solely by the pressure generated by the baby. Maternal variables also determine milk transfer as previously demonstrated in the experiment of cross-nursing reported by Woolridge et al. (1982a). The milk ejection reflex is less effective over the course of the feed, this reduction being more evident for the second compared with the first breast (Woolridge et al., 1982b). What appears predictable, if the actual difference observed between 
Table 3. Comparison of sucking parameters for human babies recorded during the first and second half of nursing episodes lasting more than $5 \mathrm{~min}$

\begin{tabular}{lcc}
\hline Sucking parameters & First half & \multicolumn{1}{c}{ Second half } \\
\hline Duration of each half (min) & $4.8 \pm 0.4$ & $4.8 \pm 0.4$ \\
Mean pressure (mmHg) & $-50.9 \pm 9.8$ & $-39.4 \pm 8.4^{*}$ \\
Maximum pressure (mmH) & $-199.1 \pm 15.8$ & $-187.2 \pm 18.4^{*}$ \\
Area under the curve (mmHg min) & $11985 \pm 1708$ & $9054 \pm 1547^{*}$ \\
Intersuck intervals (s) & $0.48 \pm 0.02$ & $0.49 \pm 0.02$ \\
$\quad$ percentil 25 & $0.62 \pm 0.03$ & $0.62 \pm 0.04$ \\
$\quad$ median & $0.83 \pm 0.04$ & $1.18 \pm 0.35$ \\
percentil 75 & $275 \pm 31$ & $218 \pm 26^{*}$ \\
\hline
\end{tabular}

Values are the mean \pm SE of 14 recordings.

*Significantly different compared with corresponding first half value $(P<0.05$, Wilcoxon matched pairs test $)$.

Table 4. Sucking parameters for human babies recorded from the first and second breast in the same nursing episode

\begin{tabular}{lcc}
\hline Sucking parameters & First breast & Second breast \\
\hline Duration of episode (min) & $5.9 \pm 0.8$ & $5.0 \pm 0.9$ \\
Mean pressure (mmHg) & $-55 \pm 9$ & $-57 \pm 13$ \\
Maximum pressure (mmHg) & $-224 \pm 17$ & $-217 \pm 18$ \\
Area under the curve (mmHg min) & $17616 \pm 3040$ & $16101 \pm 4291$ \\
Intersuck intervals (s) & & \\
$\quad$ percentil 25 & $0.61 \pm 0.07$ & $0.54 \pm 0.07$ \\
median & $0.77 \pm 0.07$ & $0.71 \pm 0.05$ \\
percentil 75 & $1.13 \pm 0.23$ & $0.86 \pm 0.06$ \\
Number of sucks & $304 \pm 65$ & $278 \pm 60$ \\
Milk transfer & & \\
$\quad$ total weight gain (g) & $63 \pm 9$ & $27 \pm 8^{*}$ \\
grams min - $_{\text {grams (mmHg min) }}^{-1}$ & $13.5 \pm 3.7$ & $5.5 \pm 1.4^{*}$ \\
grams per suck & $0.43 \pm 0.11$ & $0.18 \pm 0.04^{*}$ \\
\end{tabular}

Values are the mean \pm SE of seven recordings from each breast.

*Significantly different from corresponding first breast value $(P<0.05$, Wilcoxon matched pairs test).

both breasts holds true, is that the stimulatory function of sucking activity on mechanoreceptors is relatively greater than its feeding function in the second compared with the first breast. If this is the case, time spent by the baby on the second breast may be more related to the association between breastfeeding and amenorrhoea.

The present analysis is intended only to describe the method and demonstrate its use and is clearly insufficient to draw definite conclusions on the sucking pressure patterns and their relationship to milk transfer due to the low number of observations and the fact that babies of different ages and sex were grouped together. A more refined analysis that takes into account the most obvious variables will be possible now that a method has been established. The system described offers great potential to explore further the relationships between the physical parameters of sucking activity and the maternal responses, including milk transfer, hormonal changes and duration of lactational amenorrhoea.

C. R. Prieto was recipient of a fellowship funded by Local Arrangements Ltd, San Antonio, TX and matching donors, which is gratefully acknowledged. Part of the work was supported by Grant 94025 \#15 of The Rockefeller Foundation. We thank F. Vio for lending us the electronic balance and to the mothers who volunteered for the study.

\section{References}

Ardran GM, Kemp PH and Lind J (1958a) A cineradiographic study of bottle feeding British Journal of Radiology 31 11-22

Ardran GM, Kemp PH and Lind J (1958b) A cineradiographic study of breastfeeding British Journal of Radiology 31 156-162 
Bowen-Jones A, Thompson C and Drewett RF (1982) Milk flow and sucking rates during breast-feeding Developmental and Medical Child Neurology $\mathbf{2 4}$ 626-633

Colley JRT and Creamer B (1958) Sucking and swallowing in infants British Medical Journal $2422-423$

Díaz S, Rodríguez G, Marshall G, del Pino G, Casado ME, Miranda P, Schiappacasse V and Croxatto HB (1988) Breastfeeding pattern and the duration of lactational amenorrhea in urban Chilean women Contraception 38 $37-51$

Drewett RF and Woolridge M (1979) Sucking patterns of human babies on the breast Early Human Development 3/4 315-320

Hack M, Eastbrook MM and Robertson SS (1985) Development of sucking rhythm in preterm infants Early Human Development 11 133-140

Howie PW, McNeilly AS, Houston MJ, Cook A and Boyle H (1982) Fertility after childbirth: infant feeding patterns, basal PRL levels and postpartum ovulation Clinical Endocrinology 17 315-322
Kron RE, Stein M and Goddard KE (1962) A method of measuring sucking behavior of newborn infants Psychosomatic Medicine 25 181-191

Lucas A, Lucas PJ and Baum JD (1979) Pattern of milk flow in breast-fed infants The Lancet II 57-58

Wakerley JB, Clarke G and Summerlee AJS (1994) Milk ejection and its control. In The Physiology of Reproduction Second Edn pp 1131-1177 Eds E Knobil and JD Neill. Raven Press, Ltd, New York

Woolridge MW, Baum JD and Drewett RF (1982a) Individual patterns of milk intake during breast-feeding Early Human Development 7 265-272

Woolridge MW, How TV, Drewett RF, Rolfe P and Baum JD (1982b) The continuous measurement of milk intake at a feed in breast-fed babies Early Human Development 6 365-373 